\title{
Screening of Budesonide Nanoformulations for Treatment of Inflammatory Bowel Disease in an Inflamed 3D Cell-Culture Model
}

Fransisca Leonard ${ }^{1}$, Hussain Ali ${ }^{1}$, Eva-Maria Collnot ${ }^{1,2}$, Bart J. Crielaard ${ }^{3}$, Twan Lammers ${ }^{3}$, Gert Storm ${ }^{3}$, and Claus-Michael Lehr ${ }^{1,2}$

${ }^{1}$ Department of Biopharmaceutics and Pharmaceutical Technology, Saarland University, Saarbruecken, Germany; ${ }^{2}$ Department of Drug Delivery (DDEL), Helmholtz Institute for Pharmaceutical Research Saarland (HIPS), Helmholtz Centre for Infectious Research (HZI), Saarland University, Saarbruecken, Germany; ${ }^{3}$ Department of Pharmaceutics, Utrecht Institute for Pharmaceutical Sciences, University of Utrecht, Utrecht, The Netherlands

\begin{abstract}
Summary
Drug formulation screenings for treatment of inflammatory bowel disease (IBD) are mostly conducted in chemically induced rodent models that represent acute injury-caused inflammation instead of a chronic condition. To accurately screen drug formulations for chronic IBD, a relevant model that mimics the chronic condition in vitro is urgently needed. In an effort to reduce and potentially replace this scientifically and ethically questionable animal testing for IBD drugs, our laboratory has developed an in vitro model for the inflamed intestinal mucosa observed in chronic IBD, which allows high-throughput screening of anti-inflammatory drugs and their formulations. The in vitro model consists of intestinal epithelial cells, human blood-derived macrophages, and dendritic cells that are stimulated by the inflammatory cytokine interleukin-1 $\beta$. In this study, the model was utilized for evaluation of the efficacy and deposition of budesonide, an anti-inflammatory drug, in three different pharmaceutical formulations: (1) a free drug solution, (2) encapsulated into PLGA nanoparticles, and (3) encapsulated into liposomes. The in vitro model of the inflamed intestinal mucosa demonstrated its ability to differentiate therapeutic efficacy among the formulations while maintaining the convenience of conventional in vitro studies and adequately representing the complex pathophysiological changes observed in vivo.
\end{abstract}

Keywords: co-culture, macrophages, dendritic cells, Caco-2, inflammation model

\section{Introduction}

Inflammatory bowel diseases (IBD), encompassing ulcerative colitis (UC) and Crohn's disease (CD), are a group of chronic, remitting inflammatory diseases affecting the gastrointestinal tract. A combination of genetic predisposition and environmental factors are the primary causes of a de-regulated immune system, which results in aberrant mucosal inflammation (Baumgart and Carding, 2007). Due to the impaired integrity of the epithelial barrier, both UC and CD are characterized by increased luminal antigen uptake (Hering and Schulzke, 2009; McGuckin et al., 2009). As the disease is incurable, current therapies are directed toward induction of remission during acute episodes and prevention of relapses (Siegel, 2010). Treatment depends on the site of disease and its severity. While UC is restricted to the colon and rectum, $\mathrm{CD}$ is discontinuous and can affect any part of the gastro-intestinal tract. Common antiinflammatory approaches include 5-aminosalicylates, antibiotics, corticosteroids, immunosuppressants, anti-TNF antibodies, and other biological agents, such as cytokines (Akobeng, 2008;
Ghosh and Panaccione, 2010; Rutgeerts et al., 2009). These therapies are very promising but expensive. The standard treatment for moderate to mild cases consists of budesonide at a daily dose of $9 \mathrm{mg}$ (Greenberg et al., 1994). Although associated with weaker adverse effects compared to other corticosteroids, budesonide has demonstrated stronger adverse effects compared to the placebo due to extensive first pass metabolism and low systemic bioavailability (Löfberg et al., 1996). These adverse effects can be reduced by selectively delivering budesonide to its site of action.

The route of delivery for budesonide may be rectal, oral, or in some cases, intravenous. Rectal formulations are very effective for treatment in distal colonic areas and thus are used mainly in UC. For oral delivery, the drug compound has to resist or be protected from the acidic $\mathrm{pH}$ of the stomach and the metabolizing enzymes of the intestinal flora. Thus, orally applied antiinflammatories, such as budesonide, tend to be administered at higher doses, increasing the risk of systemic adverse effects. Many drug delivery strategies have been tried to circumvent this problem, e.g., pro-drugs, which are only activated in distal in- 
testinal areas, and coated pellets or capsules. These approaches, however, show limited bioavailability at the sites of inflammation, due primarily to rapid elimination as a result of diarrhea, which is a common symptom in IBD. Furthermore, budesonide is a hydrophobic compound and highly insoluble, demonstrating low local bioavailability.

The use of nano-sized carriers offers a promising approach to increase the bioavailability and enhance the retention time of budesonide at the target site via bioadhesion. Nanocarriers can avoid the diarrhea symptom and passively accumulate in the inflamed intestinal tissue (Hasani et al., 2009). Although the exact mechanism of accumulation is not fully understood, similarities to the enhanced permeability and retention phenomenon observed in cancerous tissues are assumed. Additionally, nanocarriers can be taken up by intestinal macrophages and dendritic cells. The encapsulated drugs then are released upon the degradation of the nanocarrier in the desired areas (Tabata et al., 1996). This passive targeting to the inflamed intestinal tissue using nanocarriers was successfully applied with different kinds of drugs (e.g., tacrolimus, rolipram, and 5-ASA) and different nanocarrier technologies by showing increased therapeutic efficacy and reduced adverse effects compared to free drug molecules (Lamprecht et al., 2001c; Rutgeerts et al., 2009; Greenberg et al., 1994). Formulation development and testing in these studies was conducted in different rodent models of colitis based on the chemical induction of an acute inflammation by dextran sodium sulfate (DSS) or tri-nitrobenzene sulfonic acid (TNBS) (Hasani et al., 2009; Lamprecht et al., 2001b).

Although the DSS and TNBS rodent models are the most widely used animal model for IBD, these models display several major disadvantages. Besides the obvious ethical issues, species differences and the methods of stimulation have led to overestimated or false results: Chemically induced models focus on the damage to the epithelial barrier and the subsequent immune reaction but fail to take into account the disregulation of the innate or adaptive immune system (Wirtz and Neurath, 2007). The disregulation of the immune system can affect both the response of certain drugs and the interaction with nano- or microcarriers. In addition, the genetic background of the animals can influence the pathogenesis. TNBS colitis in BALB/c mice presented a more Th2-type response, while a Th1 response was observed in SJL mice (Strober et al., 2002). Thus, these models lack responsiveness to IBD drugs, such as corticosteroids and 5-ASA, and are not suitable for studying the respective anti-inflammatory formulations (Murthy and Flanigan, 1999).

Disease-relevant in vitro cell culture models based on human tissues may present a promising alternative for drug formulation testing, as they allow for higher throughput and reduction of animal testing. They may also provide insight into mechanisms of action, uptake, and deposition. Such an in vitro model of the intestinal mucosa in the state of inflammation was previously established in our lab. The three-dimensional model is based on the co-culture of intestinal epithelial Caco- 2 cells with primary, blood-derived macrophages and dendritic cells as components of the intestinal innate immune system (Leonard et al., 2010). All cells in the model are of human origin, eliminating the issues with species differences often associated with the different ani- mal models. During 21 days of co-culture to allow tight barrier formation, immune cells assume an intestinal phenotype (Spottl et al., 2006). The incorporation of immunocompetent cells is crucial for the inflammation stimulation. They significantly enhance the inflammatory response after stimulation with the cytokine IL-1 $\beta$, quantified via markers of inflammation such as increased IL- 8 or TNF- $\alpha$ expression. As previously shown, the stimulated model reflects the pathophysiological changes observed at the intestinal barrier in IBD patients, such as reorganization of tight junctions, reduced barrier properties, increased immune cell activity, release of pro-inflammatory markers, and increased mucus production, providing several parameters to monitor disease status and therapeutic activity of novel antiinflammatory compounds and formulations.

In this study, two different nanocarriers were evaluated for their efficacy in inflammation treatment using the established in vitro model. One nanocarrier was generated from PLGA, an FDA approved polymer material. PLGA has been widely investigated as a scaffold for tissue regeneration and as a drug delivery vehicle in the form of implants and nano- or microsized carriers. It is prominently featured for the oral delivery route due to its biodegradability, biocompatibility, and ability to accommodate a wide range of compounds. Although the hydrophobic nature of PLGA benefits small hydrophobic compounds, the carrier has been demonstrated to be versatile by loading biomacromolecules, such as insulin, peptides, antigens, and nucleic acids (Mundargi et al., 2008). Thus PLGA nanoparticles can improve oral bioavailability of poorly soluble and permeable drugs by increased translocation and transcytosis of target compounds to their site of action in the gastrointestinal tract for IBDs and, potentially, for colorectal tumors (Dinarvand et al., 2011).

In contrast to the popularity of PLGA nanoparticles, only a few studies exploit liposomes for oral drug delivery applications. Instead, liposomes are commonly applied intravenously for a variety of treatments, from antibiotics to vaccinations (Henriksen-Lacey et al., 2011) and tumor therapies (Maruyama, 2011). Typically, PEGylated stealth formulations are employed for liposomes, reducing recognition by the reticuloendothelial system and affording prolonged circulation time in blood. The prolonged circulation half-life, together with high flexibility, allows liposomes with sizes $100-200 \mathrm{~nm}$ to extravasate through the leaky vasculature in tumors and inflamed organs and thus passively target their site of action (Maruyama, 2011; Maeda et al., 2000). The comparable passive targeting principle for malfunctioning endothelium and epithelium has not been extensively explored yet for liposomes in IBD treatment. Few groups have tried to approach the inflamed intestinal mucosa via the endothelium "backside" after intravenous delivery (Awasthi et al., 2002; Crielaard et al., 2011). Due to rapid degradation in the harsh gastric environment, liposomes are rarely applied via the luminal side of the intestine, and so far the Rubinstein lab has addressed the approach for IBD therapy after rectal application (Jubeh et al., 2006; Tirosh et al., 2009).

With the current aims (1) to rationally develop novel nanomedicines for oral and rectal IBD treatment, (2) to better understand the mechanism of nanocarrier interaction with the inflamed 
barrier, and (3) to evaluate the power of the in vitro model to differentiate between different treatments, we studied two different types of drug delivery vehicles (polymeric PLGA nanoparticles and liposomes) for glucocorticoid budesonide, comparing their anti-inflammatory activity and ability to recover epithelial barrier function in the novel $3 \mathrm{D}$ cell culture model of the inflamed intestinal mucosa. Budesonide-loaded nanoformulations were prepared, characterized, and applied to the in vitro model, using the free drug solution and blank nanocarriers as controls. IL-8 release was measured for inflammation monitoring. Transepithelial electrical resistance was monitored to observe recovery in epithelial barrier function. Furthermore, particle deposition was studied using confocal laser scanning microscopy.

\section{Materials and methods}

\subsection{Materials}

Poly(L-lactide-co-glycolide) (PLGA) (Resomer RG 503 H; inherent viscosity $0.31 \mathrm{dl} / \mathrm{g}$ ) was from Boehringer Ingelheim (Ingelheim, Germany). Budesonide was a kind gift from Boehringer Ingelheim. Polyvinylalcohol Mowiol 4-88 (PVA) was purchased from Kuraray (Frankfurt am Main, Germany). Human colon adenocarcinoma cell line Caco-2 clone C2Bbe1 was obtained from American Type Culture Collection (Rockville, MD, USA). Dulbecco's modified Eagle's medium (DMEM), fetal calf serum, and non-essential amino acids were purchased from PAA (Pasching, Austria). Trypsin/EDTA was obtained from Lonza (Basel, Switzerland). Polycarbonate transwell inserts with pore size $0.4 \mu \mathrm{m}$ were purchased from Corning Inc. (Acton, MA, USA). GM-CSF and IL-4 were obtained from R\&D Systems (Minneapolis, USA) and IL- $1 \beta$ from Promokine (Heidelberg, Germany). Ficoll Paque plus for PBMC isolation was obtained from GE Healthcare (Uppsala, Sweden) and human serum from Invitrogen (Wisconsin, USA). CBA human IL-8 Flex Set was purchased from BD Biosciences (Heidelberg, Germany). Purecol collagen was obtained from Advanced Biomatrix (Tucson, AZ, USA). 5-Fluoresceinamin (FA) and 1-ethyl-3-(3-Dimethylaminopropyl)-carbodiimide hydrochloride and all other chemicals were obtained from Sigma (St. Louis, MO, USA). All chemicals used in this study were of highest analytical grade.

\subsection{Fabrication and characterization of budesonide loaded PLGA nanoparticles}

Fluoresceinamine labeling of PLGA (FA-PLGA) was conducted as described previously (Weiss et al., 2006). From this modified polymer budesonide loaded FA-PLGA nanoparticles were prepared using the emulsion solvent evaporation method (Gurny et al., 1981; Metselaar and Storm, 2005). Briefly, $45 \mathrm{mg}$ of budesonide base was dissolved at room temperature in $15 \mathrm{ml}$ of ethylacetate containing $300 \mathrm{mg}$ of FA-PLGA. This organic phase was thereafter added via a gear pump (Gilson Minipuls, France) to an aqueous 2\% PVA solution under stirring. The emulsion was stirred for $2 \mathrm{~h}$ at room temperature before homogenizing at 13,500 rpm for $10 \mathrm{~min}$ using an Ultra Turrax T-25 (Janke and Kunkle GmbH KG, Staufen, Germany). To this, emulsion water was added drop wise under stirring to a total volume of $80 \mathrm{ml}$. The organic solvent was evaporated overnight at room temperature and precipitated nanoparticles with encapsulated drug were then separated from free budesonide by tangential flow filtration using a Vivaflow 50 cassette (Sartorius, Goettingen, Germany) with a MW cut-off of $30 \mathrm{kDa}$ and a total washing volume of $500 \mathrm{ml}$. After freeze drying in the presence of $300 \mathrm{mg}$ sorbitol as a cryoprotectant, nanoparticles were stored at $4^{\circ} \mathrm{C}$ under light protection until further use.

FA-PLGA budesonide nanoparticles were characterized for their size distribution and surface charge after 1:10 dilution with purified water using Zetasizer Nano ZS (Malvern Instruments, Herrenberg, Germany).

Encapsulation efficiency of budesonide in FA-PLGA nanoparticles was determined by dissolving $10 \mathrm{mg}$ of freeze dried particles in $10 \mathrm{ml}$ of ethyl acetate under stirring for 4 to $5 \mathrm{~h}$. Then, after complete evaporation of ethyl acetate the residue was dissolved in $5 \mathrm{ml}$ of acetonitrile:phosphate buffer, $\mathrm{pH} 3$ (4:6), for $2 \mathrm{~h}$. The solution was then filtered through a disposable syringe filter paper (CHROMAFIL GF/PET 45/25), pore size $0.45 \mu \mathrm{m}$. $1 \mathrm{ml}$ of the clear filtrate was collected and analyzed by HPLC.

The drug release was assessed by ultracentrifugation. 5-10 $\mathrm{mg}$ washed and freeze dried nanoparticles were dispersed in $5 \mathrm{ml}$ PBS of $\mathrm{pH} 6.8$ and $100 \mu 1$ aliquots of this solution were added to $1.5 \mathrm{ml}$ PBS in ultracentrifugation vials and incubated at room temperature at a constant shaking speed of $500 \mathrm{rpm}$. At particular time intervals $(1,2,4,6,24$, and $48 \mathrm{~h})$ one sample tube was centrifuged at $244,000 \mathrm{~g}$ for $25 \mathrm{~min}$ at $25^{\circ} \mathrm{C} .1 \mathrm{ml}$ of supernatant was thereafter removed from the nanoparticle pellet and quantified by HPLC.

Budesonide content in the samples was quantified via HPLC (Ulti Mate ${ }^{\circledR}$ 3000, Dionex, Germany) using a reversed phase C18 column (4.6 x $250 \mathrm{~mm}$, pore size $5 \mu \mathrm{m}$ ) (Merck KGaA, Germany). Mobile phase consisted of a mixture of phosphate buffer $(\mathrm{pH} 3)$ and acetonitrile (60:40) delivered at a flow rate of $1,900 \mathrm{ml} / \mathrm{min}$. The injection volume was $80 \mu 1$ and the retention time was $6.0 \mathrm{~min}$ for each sample with detection wavelengths of 214 and $254 \mathrm{~nm}$. The method was linear $\left(\mathrm{r}^{2}=0.9997\right)$ over a range of $20 \mathrm{ng} / \mathrm{ml}$ to $500 \mu \mathrm{g} / \mathrm{ml}$ with a limit of quantification of $347 \mathrm{ng} / \mathrm{ml}$.

\subsection{Liposome fabrication}

DPPC, PEG2000-DSPE (Lipoid), and cholesterol were weighed in a 1.85:0.15:1 ratio in a $50 \mathrm{ml}$ round bottom flask and dissolved in 5-10 ml ethanol (absolute) by heating at $50^{\circ} \mathrm{C}$. The organic phase was evaporated using rotary evaporation resulting in a dry lipid film, which was dried further under nitrogen flow for $30 \mathrm{~min}$. Due to better water solubility budesonide phosphate instead of the free base was used in the preparation of the liposomes. The drug was dissolved in reversed osmosis water at a concentration of 100 $\mathrm{mg} / \mathrm{ml}$. The lipid film and corticosteroid solution were heated to $50^{\circ} \mathrm{C}$ before adding the solution to the film. The film was hydrated for 5-10 min using a rotary evaporator without applying vacuum. After hydration, the size and polydispersity was decreased by extruding the dispersion through two 
polycarbonate filters. Starting with two extrusions through a double $200 \mathrm{~nm}$ filter and two extrusions through 200 and $100 \mathrm{~nm}$ filters, the liposomes were extruded ten times through two $100 \mathrm{~nm}$ filters. Free corticosteroid not encapsulated into the liposomes was removed by means of dialysis (MWCO $30,000 \mathrm{Da}$ ) in PBS at $4-8^{\circ} \mathrm{C}$ for $48 \mathrm{~h}$, where the PBS was refreshed regularly in order to remove all free corticosteroid. As for the PLGA, particle size and polydispersity of the liposome formulations were measured by size Dynamic Light Scattering using Zetasizer Nano ZS.

\subsection{Setting up of co-culture}

Co-culture was assembled and cultivated as described previously (Leonard et al., 2010). Briefly, dendritic cells and macrophages were isolated and differentiated from blood mononuclear cells for 7 days. 10,000 cells of each cell type were re-suspended in $80 \%$ collagen, $10 \%$ 10x RPMI, and $10 \%$ human serum and adjusted to $\mathrm{pH} 7.4$. The collagen-cell mixture was seeded on a transwell filter insert $\left(1.13 \mathrm{~cm}^{2}, 0.4 \mu \mathrm{m}\right.$ pore size $)$ and left for an hour before Caco-2 cells were seeded on top of the layer at a seeding density of 60,000 per well. The co-culture was cultivated for 21 days to allow intact Caco-2 monolayer formation. The barrier formation was monitored by Trans-Epithelial Electrical Resistance measurement (TEER) during the course of cultivation. After a tight monolayer was obtained, inflammation was induced by addition of IL- $1 \beta(10 \mathrm{ng} / \mathrm{ml}$, diluted from a stock solution prepared with $1 \%$ BSA in demineralized water) for $48 \mathrm{~h}$.

\subsection{Budesonide formulation testing}

After $48 \mathrm{~h}$ of stimulation, IL-1 $\beta$ was removed from co-culture and $100 \mu \mathrm{g} / \mathrm{ml}$ budesonide in free solution (prepared from a 100 $\mathrm{mg} / \mathrm{ml}$ stock solution of budesonide in ethanol) as well as PLGA and liposome formulations were added to the apical side of the co-culture for $4 \mathrm{~h}$, giving an effective budesonide dose of $20 \mu \mathrm{g}$ per well (Fig. 1). At the end of the incubation time budesonide formulations were removed and the co-cultures were used either for Transepithelial Electrical Resistance (TEER) value tracking and IL-8 sampling or for confocal microscopy imaging.

\subsection{IL-8 cytokine measurement}

Culture medium $(50 \mu \mathrm{l})$ was removed after 24 and $48 \mathrm{~h}$ and replaced with fresh medium. The IL- 8 protein content measurement in the cell supernatant was conducted according to the manufacturer's protocol. The supernatant was mixed with $50 \mu 1$ of CBA human IL- 8 Flex beads and left for $1 \mathrm{~h}$ before detection beads were added to the mixture and incubated for $2 \mathrm{~h}$ without light exposure. The quantification was done via a fluorescent activated cell sorter (FACS Calibur, BD Biosciences, Germany) using FCAP array v1.0.1 cytometric bead array analysis software.

\subsection{Transepithelial Electrical Resistance (TEER) measurement}

Transepithelial electrical resistance was measured with an STX2 electrode using EVOM2 (World Precision Instruments, Sarasota, FL, USA). For the measurement, the cells were placed on a pre-conditioned $37^{\circ} \mathrm{C}$ heating plate to avoid temperature shock-related TEER value fluctuation. The TEER value was obtained after subtracting the value of blank filter in the medium from the measured value.

\subsection{Confocal Laser Scanning Microscopy}

Fluorescence images were captured by a Zeiss LSM 510 confocal microscope with the software LSM 510 package. Z-stack dataset images were captured and reconstructed into threedimensional images using Volocity (Improvisions, Lexington, MA, USA) imaging software.

\subsection{Statistical analysis}

All the experiments were conducted in triplicate and repeated twice for reproducibility. The mean values, standard deviations, standard errors, and significant differences were calculated with ANOVA, Holm-Sidak test using SigmaStat 3.0 software.

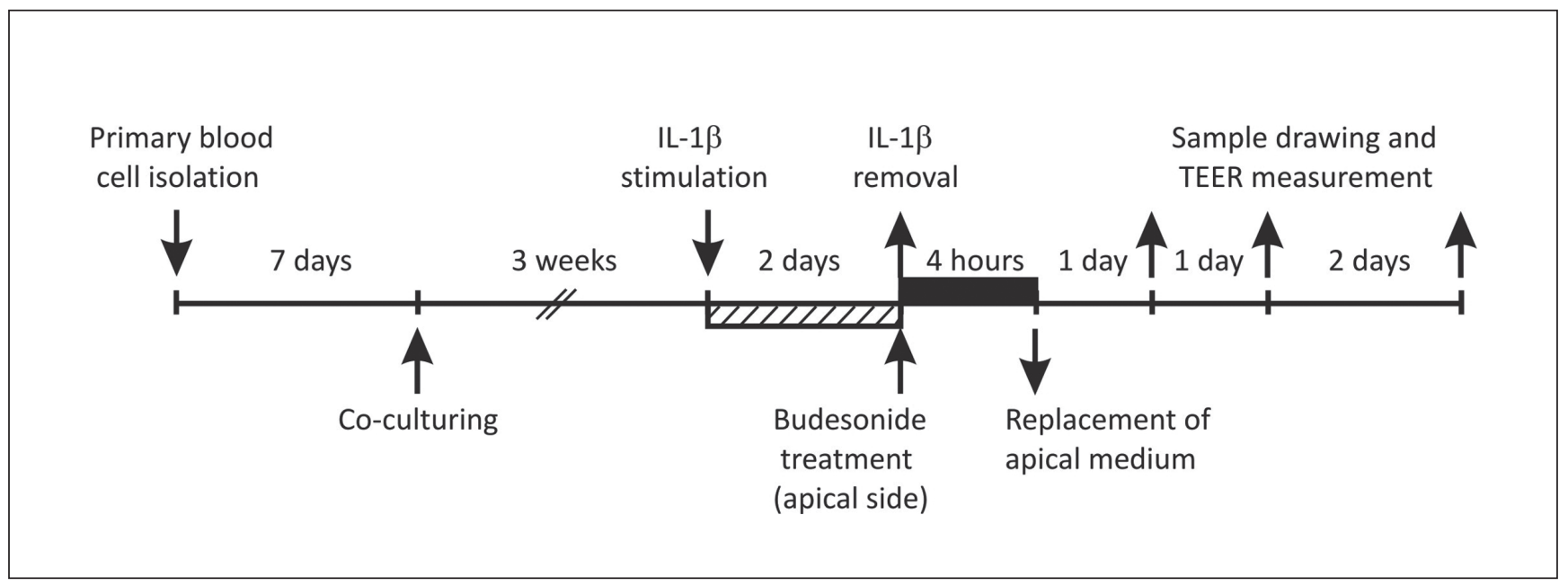

Fig. 1: Experimental timeline of setting up the in vitro model of inflamed intestinal mucosa and subsequent budesonide formulation testing 
Tab. 1: Physicochemical properties of tested formulations and blank nanocarrier controls

$\mathrm{PDI}=$ Polydispersity index, EE = Encapsulation efficiency; mean $\pm S D, n=3$

\begin{tabular}{|l|c|c|c|}
\hline Formulation & Size & PDI & E.E. (\%) \\
\hline Budesonide solution & - & - & - \\
\hline FA-PLGA-budesonide & $220 \mathrm{~nm}$ & 0.1 & 46 \\
\hline FA-PLGA - drug-free & $220 \mathrm{~nm}$ & 0.06 & - \\
\hline Liposomal budesonide & $190 \mathrm{~nm}$ & 0.0544 & 4.2 \\
\hline Liposomes - drug-free & $120 \mathrm{~nm}$ & 0.0162 & - \\
\hline
\end{tabular}

\section{Results}

\subsection{PLGA nanoparticle and liposome characterization}

Both budesonide loaded nanocarrier systems were found to be monodisperse and in the same size range of 190 to $220 \mathrm{~nm}$ (Tab. 1). Budesonide encapsulation into FA-PLGA nanoparticles did not affect the size of the polymeric particles. Similar sizes were yielded for the blank nanocarrier control with a minor variance in polydispersity, which may be attributed to batch to batch variations. A significantly smaller size was observed for drug-free liposomal formulation. In comparison with the liposomal formulation, PLGA nanoparticles demonstrated 10-times higher encapsulation efficiency at $46 \%$ of the $45 \mathrm{mg}$ drug employed in the particle preparation. The corresponding drug loading capacity for the PLGA particles was calculated at $0.07 \mathrm{mg}$ budesonide per mg FA-PLGA nanoparticles.

\subsection{TEER value monitoring}

TEER value was monitored during the course of the experiment, starting at induction of inflammation up to day 4 of budesonide treatment. It was normalized with regards to the untreated non-inflamed control, which showed stable values with normal passage to passage variations of absolute TEER in the range of 650 to $800 \Omega \times \mathrm{cm}^{2}$ (data not shown) during the entire time course of the experiment. In response to stimulation with IL-1 $\beta$, a 10 to $20 \%$ drop in TEER was observed after $48 \mathrm{~h}$, indicating successful induction of inflammation. IL-1 $\beta$ was removed at day two and cells were treated immediately with the respective budesonide formulation (the time point of treatment being indicated with an arrow in Figure 2a-c). As shown in Figure 2a, the untreated inflamed control showed slow recovery after the inflammation, indicated by the reduced TEER value of around $12 \%$ from day 2 to day 4 after stimulation. The barrier function was recovered 4 days after removal of IL- $1 \beta$. Budesonide treatment using the free drug solution acted rapidly and normalized the TEER value within the first $24 \mathrm{~h}$ after treatment (Fig. 2a). A similar result for rapid recovery is also observed from the co-culture treated with PLGA-budesonide formulation, as the TEER value reached the level of control TEER value within 24 h. Although budesonide seemed to work effectively in free solu-
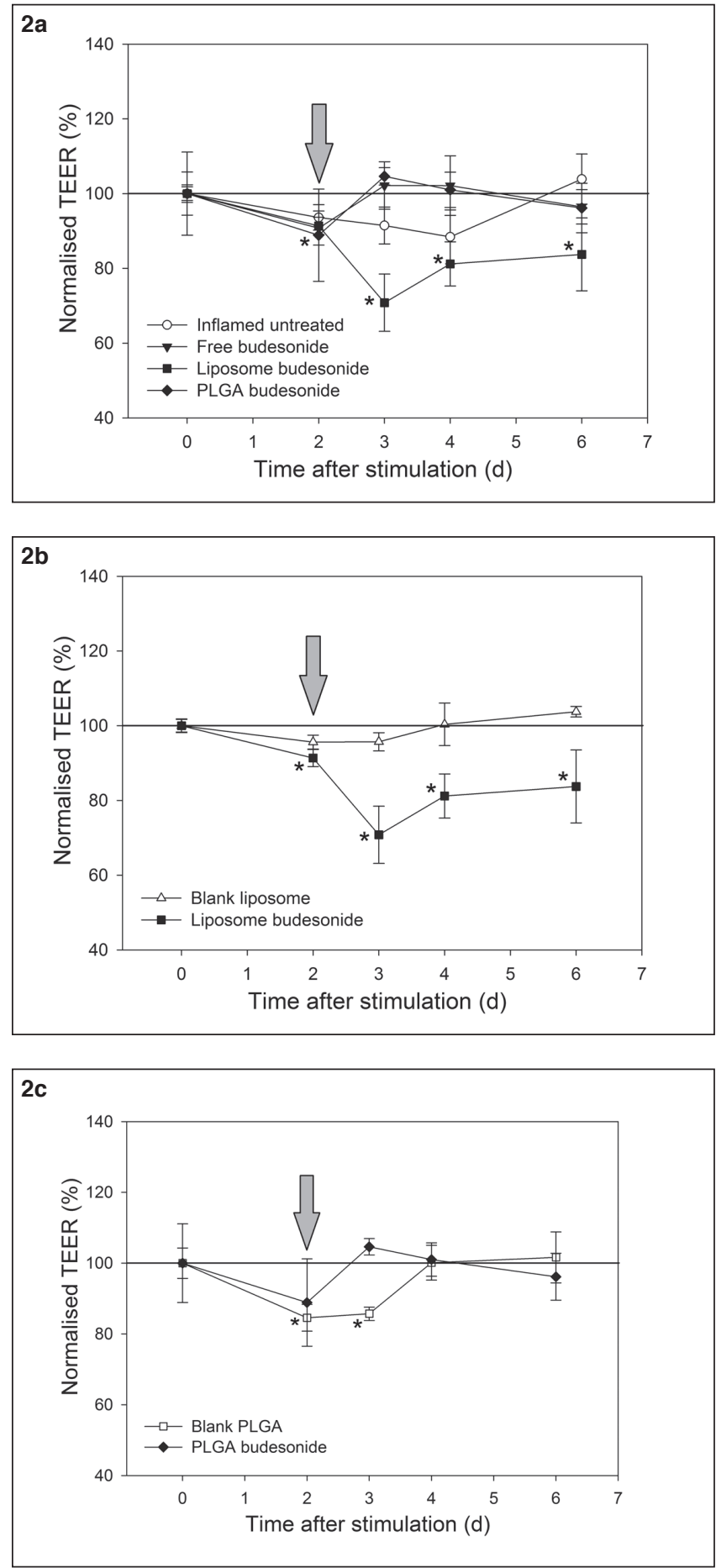

Fig. 2: TEER values of the triple co-culture model during the stimulation of inflammation and 4 days after treatment, normalized to healthy untreated control

Budesonide treatment was conducted 2 days after induction of inflammation (arrow); a) TEER changes after treatment with budesonide in free solution, liposome and PLGA formulations; b) comparison of blank liposomes and liposomal budesonide formulation; c) comparison of blank and budesonide loaded FA-PLGA nanoparticles (mean $\pm S D, n=9$, significant differences $(P<0.05)$ to non-inflamed control are indicated with *). 
tion and in PLGA particle formulation, budesonide in liposomal formulation worsened the inflammation and impaired the barrier function, as indicated by the decrease of the TEER level to $70 \%$ of the control value after the first $24 \mathrm{~h}$ of treatment. TEER values then settled around $80 \% 4$ days after treatment but did not completely recover. In comparison, blank liposomes did not have this impact on the barrier integrity (Fig. 2b) and led to a gradual recovery of the TEER value compared to the untreated inflamed control. The slow recovery of TEER value was also observed with blank PLGA nanocarriers (Fig. 2c). Treatment with both formulations without active compound recovered barrier function $48 \mathrm{~h}$ after treatment, which was slower than the formulations with budesonide but faster than the untreated inflamed control.

\subsection{IL-8 release rate}

As another marker of inflammation, the IL-8 release of the cells under budesonide treatment was measured. The healthy untreated control released around $8 \mathrm{pg} / \mathrm{ml} * \mathrm{~h}$ IL-8 (data not shown). Upon stimulation with IL- $1 \beta$, increased IL- 8 release was observed in all experiments with release rates ranging between 20-25 pg/ml*h $48 \mathrm{~h}$ after induction of inflammation (Fig. 3). The variance in IL-8 release can be attributed to normal variations among different triple culture isolations. At this point, IL- $1 \beta$ was removed from the cell culture and cells were treated with the respective budesonide formulations at a budesonide concentration of $100 \mu \mathrm{g} / \mathrm{ml}$ or blank carrier/medium control for $4 \mathrm{~h}$. Upon removal of IL-1 $\beta$ the medium control showed slight self-healing effects as the IL-8 release dropped to around
$40 \%$ from the initial inflammation level but was twice as high as the healthy control (Fig. 3). Treatment with free budesonide reduced the IL- 8 production to $13 \%$ of the initial inflamed value during the course of the first day, which is similar to the levels of the healthy control. IL-8 production increased again in the following days, reaching $36 \%$ of the initial inflamed value. The two liposome formulations, with or without budesonide, induced the highest IL-8 release rate. In the cells treated with blank liposomes, IL-8 production was reduced to $38 \%$ of initial value after one day, corresponding to the values from the nontreated medium control. However, the relapse over the next few days was stronger as IL-8 levels reached $75-80 \%$ of the initial value after 2-4 days. Budesonide-loaded liposomes increased production of IL- 8 to $124 \%$ and $156 \%$ of the initial value after 24 and 48 h, respectively, reaching 3 times higher IL-8 levels than the starting values after 4 days. PLGA formulation seemed to be effective in reducing IL-8 release, as PLGA-budesonide particles led to a $16 \%$ decrease of IL- 8 during the first 2 days. This decrease indicates a prolonged effect of PLGA-budesonide compared to the free budesonide formulation, as IL- 8 release remained at low levels of $19 \%$ compared to the healthy control after 4 days of treatment. The blank PLGA particles also reduced the IL-8 level to a fluctuating value of around 30 to $50 \%$ of the initial value.

\subsection{Deposition of drug carrier systems}

For confocal laser scanning microscopy experiments, nanocarrier systems were fluorescently labeled by covalent coupling of fluorescein to PLGA (Weiss et al., 2006) and by rhodamine

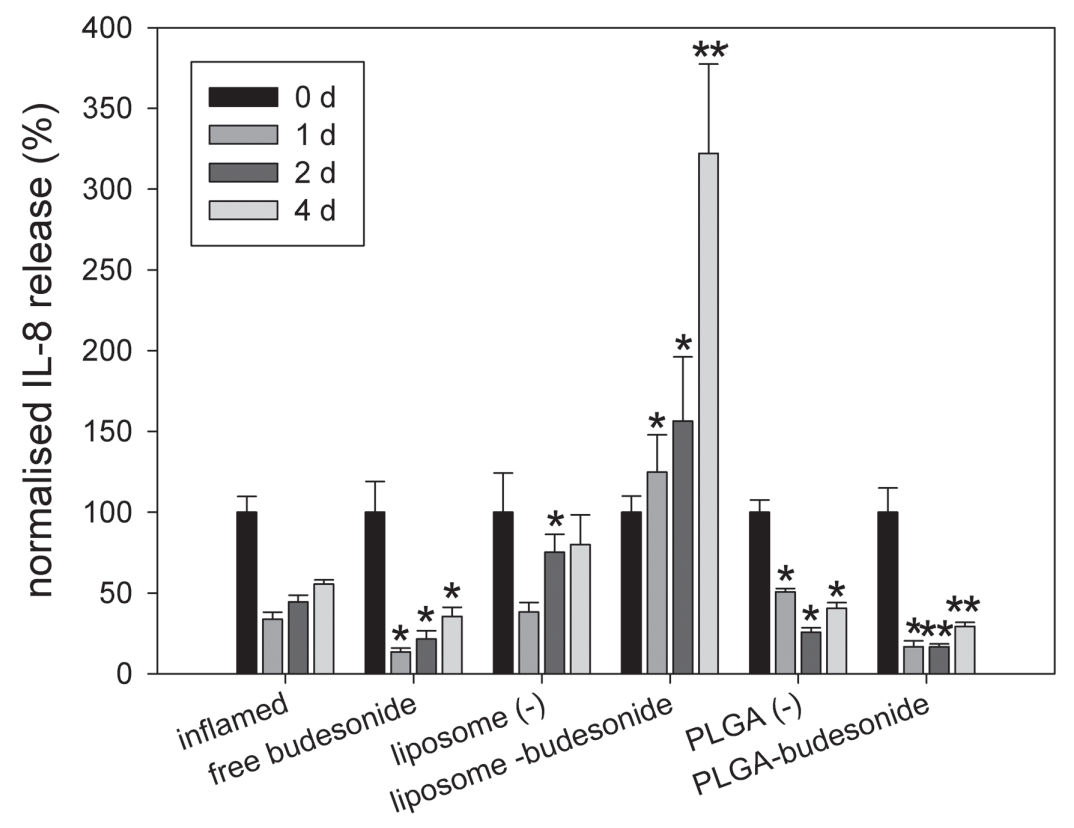

Fig. 3: Normalized IL-8 release rate of triple co-culture model after induction of inflammation and treatment effects of various budesonide formulations and blank nanocarrier controls Mean $\pm S D, n=9,{ }^{*} P<0.05,{ }^{* *} P<0.01$ vs. healthy untreated control 
coupling to the liposomal lipid. For easier recognition, the red rhodamine signal was translated to green signal in the images displayed. The fluorescence microscopy pictures revealed the deposition of liposomes and PLGA-budesonide particles throughout the healthy and inflamed in vitro model. In both the healthy and inflamed triple culture setup, only low amounts of PLGA-budesonide particles were observed to adhere to the apical surface (Fig. 4a,c). In contrast, in the inflamed model, the particles were found in the junctional space of the monolayer and no co-localization with immunocompetent cells was observed (Fig. 4c). The liposomes were deposited on the surface and, to some extent, into the basolateral layer of the healthy model (Fig. 4b). A deeper penetration of the liposomes was observed in the inflamed model, in which the fluorescent signals were primarily found in the basolateral side of the model (Fig. $4 d)$. In both healthy and inflamed models, some but not exclusively all rhodamine signals from the liposome were found in co-localization with the auto-fluorescence signal of immunocompetent cells (Fig. 4b,d). Furthermore, the rhodamine signal appeared more diffuse and spread out, indicating a break down or processing of the liposomes.

\section{Discussion}

As reported previously, a novel in vitro model of the inflamed intestinal mucosa was established and characterized in our laboratory (Leonard et al., 2010). To further evaluate the power
$4 a$
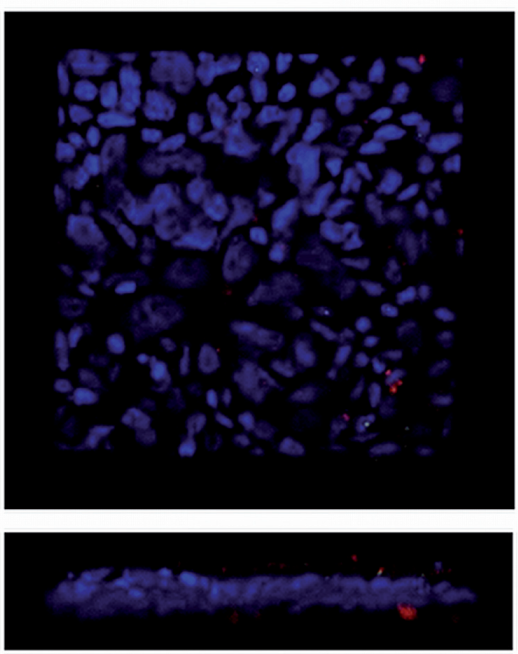

$4 c$

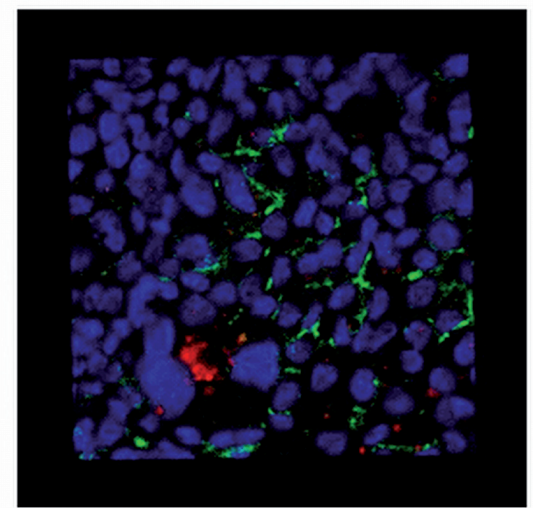

Q.
$4 b$
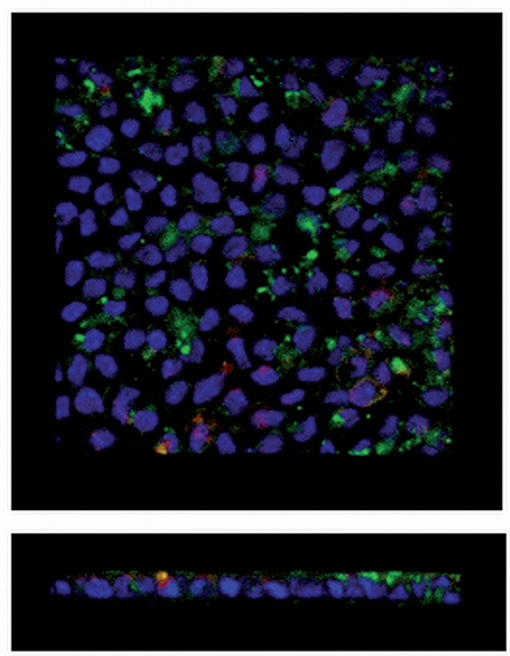

4d
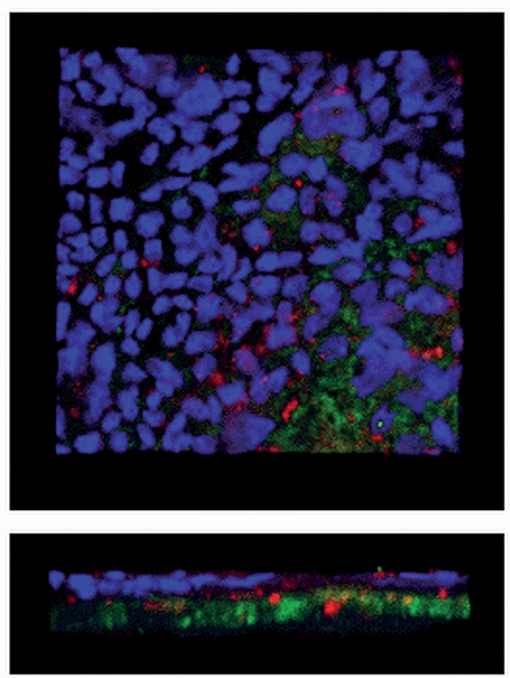

Fig. 4: Deposition of budesonide loaded FA-PLGA nanoparticles (a\&c) and liposomal budesonide (b\&d) in the triple co-culture model of the intestinal mucosa in non-inflamed state (a\&b) and after induction of inflammation by IL-1 $\beta$ (c\&d) as observed by confocal laser scanning microscopy

Blue signal: Caco-2 cell nuclei stained with DAPI; red signal: autofluorescence of immunocompetent cells; green signal: fluorescence labeled nanoformulations (red) 
of the in vitro model in the context of pharmacology (formulation development and drug delivery research), two different carrier systems (a PLGA polymeric nanoparticle system and a liposomal system) loaded with the glucocorticoid budesonide were evaluated in the system. Both therapeutic efficacy and the mechanism of uptake and action for the two nanocarrier systems were tested in the in vitro model.

Nanocarrier systems have been extensively used in drug delivery applications to control release and actively or passively target the site of action. With regards to IBD treatments, nanocarriers were previously shown in different animal colitis models to accumulate passively in inflamed intestinal areas, forming a drug depot for encapsulated compounds and thus improving anti-inflammatory therapy and reducing systemic adverse effects (Lamprecht et al., 2001a). Both PLGA polymeric nanoparticles and liposomes are widely used in the field of nanomedicine for their easy-to-handle manner and their biodegradability. PLGA polymers are degraded to their components in the body through hydrolysis of the ester-bonds to lactic acid and glycolic acid. These molecules are further metabolized in the citric acid cycle, contributing to their biocompatibility and low toxicity. PLGA nanocarriers have previously been investigated for application in IBD as delivery systems for 5-aminosalicylic acid and tacrolimus and after chitosan surface modification for localized delivery of nuclear factor kappa decoy oligonucleotide (Tahara et al., 2011). To increase their stability in low $\mathrm{pH}$ and target the drug release to the distal ileum and colon, PLGA can also be blended with Eudragit S100 (Gurny et al., 1981; Makhlof et al., 2009).

Although some studies showed the potential of liposomes for oral drug delivery, their stability in the low $\mathrm{pH}$ and enzyme-rich environment of the gastrointestinal tract presents a significant issue. To target the inflamed intestinal mucosa from the luminal side, liposomes have to be applied rectally as enemas, avoiding the stressful gut passage and issues of stability and coalescence. The intravenous pathway is more common for liposome applications, in which they passively accumulate via endothelium fenestrations in inflamed areas. Stealth liposomal formulations as employed in this study are known to escape opsonization and uptake by the reticuloendothelial system, which increases their circulation half-life and extravasation likelihood (Immordino et al., 2006). Both carrier systems are used as "Trojan horses" for poorly soluble hydrophobic compounds, such as budesonide.

In this study, budesonide was incorporated into the hydrophobic core of the PLGA nanoparticles and within the liposomes, thus increasing its apparent solubility and bioavailability. The encapsulation efficiency for budesonide in liposomes was about 10-times lower than in FA-PLGA nanoparticles, which presented an ideal matrix for incorporation of small hydrophobic compounds with an encapsulation efficiency of $46 \%$ and a drug loading rate of $7 \%$. It is to be noted that the fluorescence labeling of the polymer by covalent linking of fluorescein to the 50:50 PLGA polymer for detection in the confocal microscopy study led to a more hydrophilic derivative and reduced the drug loading capacity compared to the native polymer. The native polymer was found to accommodate up to $14 \%$ of budesonide drug in line with previous glucocorticoid PLGA formulations (data not shown). For the in vitro studies, the differences in drug loading between the two evaluated formulations was compensated by normalizing the amount of the respective formulation. The budesonide concentration used was $100 \mu \mathrm{g} / \mathrm{ml}$ to reach a comparable effect in the order of magnitude of the daily dosage of Entocort ${ }^{\circledR} \mathrm{EC}$, which results in a concentration of 36 $\mu \mathrm{g} / \mathrm{ml}$ in intestinal fluid. The incubation time in the model was $4 \mathrm{~h}$ to approximate drug retention time in the intestine of IBD patients.

At the site of action, drug release from non-coated PLGA nanoparticles is a two-step process with an initial burst release of drug adsorbed to the particle surface or bound to the outer layer of the particle followed by a slower, continuous release mediated by a combination of compound diffusion through nanopores and -channels and polymer degradation (Allison, 2008). Under sink conditions, the budesonide burst release from FA-PLGA nanoparticles in this study was found to be $42 \%$ in the first $4 \mathrm{~h}$, leaving almost $60 \%$ of encapsulated drug to form a drug depot at the target site (supplementary file A; www.altex-edition.org). Although it was previously known that stealth liposomes escape the reticuloendothelial system when delivered from the blood side, the liposomes in our study seemed to be internalized and degraded by activated intestinal immunocompetent cells, leading to an area-concentrated burst release after application from the apical side of the intestinal model (Metselaar and Storm, 2005).

In our study, TEER values were decreased upon induction of inflammation in the co-culture model. Epithelial barrier integrity was disrupted due to tight junctional reorganization. Free budesonide showed a strong anti-inflammatory effect by immediately recovering the TEER value and reducing the IL8 release in the model (Fig. 2a). PLGA-budesonide nanoparticles showed a similar anti-inflammatory effect. The results from IL-8 analysis, however, revealed the longevity of the PLGA treatment effect (Fig. 3). In the cells treated with free budesonide solution, we observed an immediate decrease of IL- 8 production within the first $24 \mathrm{~h}$ of treatment. The effect did not last long, and the release rate rebounded and increased after the second day to fourth day, which fits with the in vivo dosing scheme of free budesonide that is renewed daily (Greenberg et al., 1994). Meanwhile, the PLGA-budesonide dispersion reduced the IL-8 release levels almost to the levels of the healthy control and maintained them throughout the experimental duration of 4 days (Fig. 3).

A slight carrier effect was observed in the control experiments, as cells treated with drug-free PLGA nanoparticles, as well as blank liposomes, showed a faster recovery of barrier properties compared to control (Fig. 2b,c). In addition, blank PLGA nanoparticles significantly reduced IL-8 inflammation marker levels after $24 \mathrm{~h}$ and $48 \mathrm{~h}$ of treatment (Fig. 3). This phenomenon may be related to blank particle interaction with immune cells in the triple culture, leading to a preoccupation of the immune system with this new stimulus. However, only low levels of probing and internalization of PLGA nanocarriers by immunocompetent cells were observed by confocal images. Alternatively, an adsorption of soluble signaling parameters in the inflammatory cascade of IL- 8 to the particle surface might explain the findings. 
The proposed hypothesis of a prolonged activity of PLGAbudesonide nanoparticles due to depot formation and prolonged budesonide release is supported by the findings from the confocal microscopy study. Epithelial barrier function was disrupted and slightly loosened up in the inflamed model, allowing the PLGA nanoparticles to accumulate in the space between the cells while not translocating to the basolateral side. This effect was not observed in the healthy model where hardly any particles adhered to the apical side or accumulated in the intercellular space. The PLGA nanoparticles at $220 \mathrm{~nm}$ size were retained in the upper region of the tight junctions, since they are bigger than the tight junctional pore of 58 to $104 \mathrm{~nm}$ (Linnankoski et al., 2010). The inflammation in the cell model leads to a broadening of intercellular space in which the nanoparticles are trapped, while not allowing diffusion to the basolateral side. This differs from our previous observation with Fluoresbrite ${ }^{\circledR}$ nanoparticles, in which the particles were deposited on the top of the monolayer or internalized by activated immunocompetent cells (Leonard et al., 2010). Those particles were, however, rigid polystyrene particles and not biodegradable. Therefore, they were developed as model particles without concerns about drug delivery properties.

As reported in several publications, fluorescent dye in labeled nanocarriers may leak or be dissociated from the nanocarriers (Suh et al., 1998; Pietzonka et al., 2002), giving erroneous particle uptake and distribution data. The dissociation of fluorescein is unlikely in this study, as the dye was covalently bound to the PLGA polymers and previously shown not to diffuse out of the PLGA nanoparticles (Metselaar and Storm, 2005).

In contrast to the PLGA nanoparticles, the liposomal formulation did worsen the inflammation by disrupting the barrier integrity and increasing the IL-8 release of the cells (Fig. 2a,b). The resulting epithelial defects are clearly detectable as large areas without DAPI stained nuclei by confocal imaging of the liposome treated 3D model (Fig. 4d). The loosening and rupture of the epithelial barrier resulted in transversing liposomes, which were then directly exposed to immunocompetent cells on the basolateral side (Fig. 4d). In our study, the rhodamine dye was incorporated into the liposome via covalent binding to the lipid (1,2-dipalmitoyl-sn-glycero-3-phosphoethanolaine$\mathrm{N}$-(Lissamine rhodamine B Sulfonyl)). This coupling ensured that the fluorescence signal detected is mostly from the lipid. It does not, however, warrant the integrity of the liposomes. From the diffuse signal in the confocal images and the deposition of the fluorescence signal, we may gather that the lipids may have been dissociated and the liposome itself broken down. This effect is not associated with the liposome carrier itself but rather with the specific liposomal budesonide formulation. The blank liposomes slightly increased the IL-8 release. In contrast, the budesonide liposome formulation induced a significantly higher IL-8 release compared to the initial release in inflamed cells (Fig. 3). Therefore, the toxicity is likely not associated to a formulation component or the liposome concentration (which is 10-fold higher compared to the PLGA nanoparticles to compensate for the lower loading capacity). The toxicity is probably caused by the sudden budesonide release on the basolateral side as a consequence of liposome uptake and disruption by phago- cytic cells. The evaluated liposomal budesonide formulation at the concentration used in this study is thus not applicable for IBD treatment.

These results are in agreement with a previous study, in which a similar glucocorticoid (dexamethasone) in liposomal formulation seemed to aggravate the disease in a DSS mouse model of colitis. In contrast, the same dexamethasone formulation improved the condition in multiple sclerosis animal models (Crielaard et al., 2011). The authors hypothesize that preferential uptake into tissue macrophages and shifting of macrophages may account for the differences observed. In the intestine, the M1 macrophage phenotype may be essential to keep up the innate immune answer, preventing pathogen invasion across the barrier and, at the same time, tolerating commensal intestinal flora. However, direct uptake of glucocorticoid tends to shift the macrophage population to a more anti-inflammatory M2 characteristic, leading to more aberrant activation of the immune system. Thus anti-inflammatory therapy in epithelial and dendritic cells may be beneficial for IBD treatment, while interference with macrophage function should be avoided. For successful application in IBD treatment, liposomes not only have to withstand diverse physiological conditions found in the GI tract but also have to be specifically directed to inflamed areas of the intestine. Jubeh et al. reported the significance of charge properties for the liposome adhesion behavior (Jubeh et al., 2004). Anionic liposomes were found augmented in inflamed areas of rat intestine, while cationic or neutral liposomes were accumulating in healthy tissue. The negative charge of the liposome used in this study may promote the adhesion to inflamed tissue, but the uptake by phagocytic macrophages seemed particularly to worsen the inflammation.

In summary, the PLGA-budesonide formulation was found, in this study, to be superior to both free budesonide solution and liposome formulation. As the first drug-loaded nanocarriers tested in the triple co-culture model, the particles showed advantageous properties and good efficacy for recovery from inflammation, as indicated by TEER value and pro-inflammatory protein release. This formulation also showed evidence of a depot effect for budesonide release. Another advantage is the higher encapsulation efficiency of PLGA particles in comparison to the liposome. This avoids a higher loss of budesonide in the generation of the formulation. The PLGA particles did not adhere to the healthy model, nor were they translocated to the basolateral side, showing their ability to avoid preemptive absorption in the upper, non-affected parts of the gastro-intestinal tract and thus systemic exposure. Instead, they specifically adhere to the more mucus-rich and acidic inflamed model tissue also commonly found in IBD patients (Press et al., 1998). Furthermore, the loosening of tight junctional complexes increases their chance to reach and accumulate in the targeted inflammation area.

Budesonide loaded-liposomes at the concentration studied were proven to be toxic to the inflamed tissue, which was likely due to the fast release of budesonide after uptake by immunocompetent cells. The effect of this dose dump is thought to be more pronounced for the liposomes than for the free drug solution, as much of the budesonide dose is localized to the immune 
cells instead of being equally distributed among all three cell types in the triple culture. In conclusion, a lower dosage of liposomes or lower budesonide loading might be preferential for localized IBD treatment.

Several cell culture models incorporating immunocompetent cells, such as macrophages or B-cells, to simulate intestinal tissue have been developed (Tanoue et al., 2008; des Rieux et al., 2007; Spottl et al., 2006). In our model, the inflammatory response is promoted by addition of a pro-inflammatory cytokine to the triple culture of epithelial and immunocompetent cells to mimic the inflammation in IBD. This model is able to reflect the long-term recovery process after controlled inflammation, while it can be used to study mechanisms of carrier uptake in a complex system. Macrophages and dendritic cells were in direct contact with the epithelial cells, allowing the monitoring and tracking of carrier deposition and further processing in the inflamed intestinal tissue.

The superiority of the triple co-culture model compared to conventional cell culture models (Leonard et al. 2010) has been underlined in this study. Different read-out parameters could be used to evaluate the treatment efficacy, and the experimental setup gives a better representation of pathophysiological changes in IBD compared to Caco-2 cells alone. In particular, the involvement of immune cells in the inflammatory response is essential, giving significantly higher inflammatory marker release compared to the respective monocultures. This interplay seems to exist between all three cell types in the co-culture model, as only the presence of both dendritic cells and macrophages synergistically enhanced the strength of the immune answer, as shown previously (Leonard et al. 2010). Also, a preferential uptake of nanoparticles and other foreign objects into immune cells was observed. As we are not able to differentiate between both immune cell types by direct antibody staining due to embedding in the collagen layer, it is not possible to state which immune cell does the majority of nanoparticle or liposome processing. However, previous studies in a triple culture model of the alveolar mucosa found a preferential uptake by monocyte-derived macrophages, which then passed on the particulate cargo to the dendritic cells for further antigen processing and induction of an immune answer (Blank et al., 2011).

In conclusion, testing in this advanced in vitro model should allow us to study the drug and formulation effect on the epithelial barrier as well as on the innate immune system. The model was found to be powerful for the screening of suitable anti-inflammatory formulations and drugs as a pre-stage for in vivo animal studies. Thus the number of animal tests can be reduced, lessening the ethical burden and speeding up the screening process and the development of novel IBD therapies.

\section{References}

Akobeng, A. K. (2008). Crohn's disease: Current treatment options. Arch Dis Child 93, 787-792.

Allison, S. D. (2008). Analysis of initial burst in PLGA microparticles. Expert Opin Drug Del 5, 615-628.

Awasthi, V. D., Goins, B., Klipper, R., et al. (2002). Accumulation of PEG-liposomes in the inflamed colon of rats: Poten- tial for therapeutic and diagnostic targeting of inflammatory bowel diseases. J Drug Target 10, 419-427.

Baumgart, D. C. and Carding, S. R. (2007). Inflammatory bowel disease: cause and immunobiology. Lancet 369, 1627-1640.

Blank, F., Wehrli, M., Lehmann, A., et al. (2011). Macrophages and dendritic cells express tight junction proteins and exchange particles in an in vitro model of the human airway wall. Immunobiology 216, 86-95.

Crielaard, B. J., Lammers, T., Morgan, M. E., et al. (2011). Macrophages and liposomes in inflammatory disease: Friends or foes? Int J Pharm 416, 499-506.

des Rieux, A., Fievez, V., Théate, I., et al. (2007). An improved in vitro model of human intestinal follicle-associated epithelium to study nanoparticle transport by M cells. Eur J Pharm Sci 30, 380-391.

Dinarvand, R., Sepehri, N., Manoochehri, S., et al. (2011). Polylactide-co-glycolide nanoparticles for controlled delivery of anticancer agents. Int J Nanomedicine 6, 877-895.

Ghosh, S. and Panaccione, R. (2010). Review: Anti-adhesion molecule therapy for inflammatory bowel disease. Therap Adv Gastroenterol 3, 239-258.

Greenberg, G. R., Feagan, B. G., Martin, F., et al. (1994). Oral budesonide for active Crohn's disease. N Engl J Med 331, 836-841

Gurny, R., Peppas, N. A., Harrington, D. D., et al. (1981). Development of biodegradable and injectable latices for controlled release of potent drugs. Drug Dev Ind Pharm 7, 1-25.

Hasani, S., Pellequer, Y., and Lamprecht, A. (2009). Selective adhesion of nanoparticles to inflamed tissue in gastric ulcers. Pharm Res 26, 1149-1154.

Henriksen-Lacey, M., Korsholm, K. S., Andersen, P., et al. (2011). Liposomal vaccine delivery systems. Expert Opin Drug Del 8, 505-519.

Hering, N. A. and Schulzke, J. D. (2009). Therapeutic options to modulate barrier defects in inflammatory bowel disease. Dig Dis 27, 450-454.

Immordino, M. L., Dosio, F., and Cattel, L. (2006). Stealth liposomes: review of the basic science, rationale, and clinical applications, existing and potential. Int J Nanomedicine 1 , 297-315.

Jubeh, T. T., Barenholz, Y., and Rubinstein, A. (2004). Differential adhesion of normal and inflamed rat colonic mucosa by charged liposomes. Pharm Res 21, 447-453.

Jubeh, T. T., Nadler-Milbauer, M., Barenholz, Y., et al. (2006). Local treatment of experimental colitis in the rat by negatively charged liposomes of catalase, TMN and SOD. J Drug Target 14, 155-163.

Lamprecht, A., Schafer, U., and Lehr, C. M. (2001a). Size dependency of microparticle deposition to the inflamed colon in inflammatory bowel disease: in-vivo results from rat. J Control Release 72, 235-237.

Lamprecht, A., Schafer, U., and Lehr, C. M. (2001b). Size-dependent bioadhesion of micro- and nanoparticulate carriers to the inflamed colonic mucosa. Pharm Res 18, 788-793.

Lamprecht, A., Ubrich, N., Yamamoto, H., et al. (2001c). Biodegradable nanoparticles for targeted drug delivery in treatment of inflammatory bowel disease. J Pharmacol Exp Ther 
299, 775-781.

Leonard, F., Collnot, E. M., and Lehr, C. M. (2010). A three-dimensional coculture of enterocytes, monocytes and dendritic cells to model inflamed intestinal mucosa in vitro. Mol Pharm 7, 2103-2119.

Linnankoski, J., Mäkelä, J., Palmgren, J., et al. (2010). Paracellular porosity and pore size of the human intestinal epithelium in tissue and cell culture models. J Pharm Sci 99 , 2166-2175.

Löfberg, R., Rutgeerts, P., Malchow, H., et al. (1996). Budesonide prolongs time to relapse in ileal and ileocaecal Crohn's disease. A placebo controlled one year study. Gut 39, 82-86.

Maeda, H., Wu, J., Sawa, T., et al. (2000). Tumor vascular permeability and the EPR effect in macromolecular therapeutics: A review. J Control Release 65, 271-284.

Makhlof, A., Tozuka, Y., and Takeuchi, H. (2009). pH-Sensitive nanospheres for colon-specific drug delivery in experimentally induced colitis rat model. Eur J Pharm Biopharm 72, 1-8.

Maruyama, K. (2011). Intracellular targeting delivery of liposomal drugs to solid tumors based on EPR effects. Adv Drug Deliv Rev 63, 161-169.

McGuckin, M. A., Eri, R., Simms, L. A., et al. (2009). Intestinal barrier dysfunction in inflammatory bowel diseases. Inflamm Bowel Dis 15, 100-113.

Metselaar, J. M. and Storm, G. (2005). Liposomes in the treatment of inflammatory disorders. Expert Opin Drug Del 2, 465-476.

Mundargi, R. C., Babu, V. R., Rangaswamy, V., et al. (2008). Nano/micro technologies for delivering macromolecular therapeutics using poly(d,l-lactide-co-glycolide) and its derivatives. J Control Release 125, 193-209.

Murthy, S. and Flanigan, A. (1999). Animal Models of inflammatory bowel disease. In D. W. Morgan and L. A. Marshall (eds.), In Vivo Models of Inflammation. Basel, Switzerland: Birkhäuser Verlag AG.

Pietzonka, P., Rothen-Rutishauser, B., Langguth, P., et al. (2002). Transfer of lipophilic markers from PLGA and polystyrene nanoparticles to Caco-2 monolayers mimics particle uptake. Pharm Res 19, 595-601.

Press, A. G., Hauptmann, I. A., Hauptmann, L., et al. (1998). Gastrointestinal $\mathrm{pH}$ profiles in patients with inflammatory bowel disease. Aliment Pharmacol Ther 12, 673-678.

Rutgeerts, P., Vermeire, S., and Van Assche, G. (2009). Biological therapies for inflammatory bowel diseases. Gastroenterology 136, 1182-1197.

Siegel, C. A. (2010). What options do we have for induction therapy for Crohn's disease? Dig Dis 28, 543-547.

Spottl, T., Hausmann, M., Gunckel, M., et al. (2006). A new organotypic model to study cell interactions in the intestinal mucosa. Eur J Gastroenterol Hepatol 18, 901-909.

Strober, W., Fuss, I. J., and Blumberg, R. S. (2002). The immunology of mucosal models of inflammation. Annu Rev Immunol 20, 495-549.

Suh, H., Jeong, B., Liu, F., et al. (1998). Cellular uptake study of biodegradable nanoparticles in vascular smooth muscle cells. Pharm Res 15, 1495-1498.

Tabata, Y., Inoue, Y., and Ikada, Y. (1996). Size effect on systemic and mucosal immune responses induced by oral administration of biodegradable microspheres. Vaccine 14, 16771685.

Tahara, K., Samura, S., Tsuji, K., et al. (2011). Oral nuclear factor- $x \mathrm{~B}$ decoy oligonucleotides delivery system with chitosan modified poly(d,1-lactide-co-glycolide) nanospheres for inflammatory bowel disease. Biomaterials 32, 870-878.

Tanoue, T., Nishitani, Y., Kanazawa, K., et al. (2008). In vitro model to estimate gut inflammation using co-cultured Caco-2 and RAW264.7 cells. Biochem Biophys Res Commun 374, 565-569.

Tirosh, B., Khatib, N., Barenholz, Y., et al. (2009). Transferrin as a luminal target for negatively charged liposomes in the inflamed colonic mucosa. Mol Pharm 6, 1083-1091.

Weiss, B., Schaefer, U. F., Zapp, J., et al. (2006). Nanoparticles made of fluorescence-labelled Poly(L-lactide-co-glycolide): preparation, stability, and biocompatibility. J Nanosci Nanotechnol 6, 3048-3056.

Wirtz, S. and Neurath, M. F. (2007). Mouse models of inflammatory bowel disease. Adv Drug Deliv Rev 59, 1073-1083.

\section{Acknowledgements}

This research has been conducted with financial support of the European Union Sixth Framework Program Integrated Project MEDITRANS Contract. No. 026668. We thank Hamsa Jaganathan for editing the manuscript.

\section{Correspondence to}

Eva-Maria Collnot, $\mathrm{PhD}$

Department of Drug Delivery (DDEL)

Helmholtz Institute for Pharmaceutical

Research Saarland (HIPS)

Saarland University

Campus A4.1

66123 Saarbrücken

Germany

Phone: +49 6813022939

Fax: +49 6813024677

e-mail: eva-maria.collnot@helmholtz-hzi.de, 